\title{
Immersive Technology: Towards a Kineikonic Dialogism in Challenging the Myth of the Frame Gregg S. Lloren
}

This paper contributes to the introductory study on the sociocultural impact of immersive technology or $\operatorname{ImT}$, in the form of $360^{\circ}$ capture and virtual reality projection. A young technology in the field of visual language, ImT challenges the supremacy of the frame in cinematic mediums-TV, video, film—and, in effect, introduces new notions in visual grammar of the multimodality of moving images, aka the kineikonic mode of media theorist Andrew Burn (2013). Using the dialogic system of Mikhail Bakhtin, this paper situates the place of immersive technology in the historiography of visual language, from the proscenium of the classical theater to cinema, and to virtual reality. In doing so, this study is able to demonstrate how immersive technology becomes the newest expression of mankind's linguistic resolve to transcend its physical limitations in the field of communication, information production and consumption, knowledge transfer, and dissemination of cultures.

Keywords: digital culture, kineikonic dialogism, virtual reality, immersive technology, $360^{\circ}$ video environment

\section{Overview}

This essay constitutes my preliminary investigations on the language of immersive technology ${ }^{1}$, under the confines of the multimodality of moving images, what Andrew Burn (2013) calls the kineikonic mode. In this study, the focus has been extended from the single camera image capture to the $360^{\circ}$ format ${ }^{2}$. The interest that spawned this academic inquiry comes from the notion that the study on $360^{\circ}$ image capture and projection, as a very young technology, can never be ever complete without alluding to its predecessors. In other words, there is a need to establish a dialogic in visual cultures that would demonstrate how immersive technology fits in the historiography of moving image texts. As Dina Spicheva (2014) put it:

All the manifestations of culture are combined in this digital Universe which merges the past, the present, and future manifestations of the communicative thought in a gigantic historical supertext. (p. 82) 
If the invention of immersive technology is the next obvious advancement in the history of kineikonic technologies such as cinema and television, does it simply fit in a series of technologies that could be replaced by future inventions within this spectrum of technologies? Or can it be approached as an expression of visual culture that speaks of man's effort to transcend the limitation of physical embodiment?

Answering the aforementioned complex questions entails refocusing numerous developments in my study of visual language. The framework I am fostering in my investigation of the grammar of visual language now not only largely includes concerns pertaining to the advances of communication and media technology and how new perspectives that come with these advances affect media production and consumption, but also caters to the introduction of a new genre, that is, immersive virtual reality, in visual language; reestablishes thehistorical relationships and intermediary concepts between media formats and their implications to the future developments in the field of visual inscriptions, either static or moving images; and reconfigures the sociocultural dimensions in the introduction of immersive technologies $-360^{\circ}$ camera capture and virtual reality projection-as they impact visual practice and culture, among other things - the list of concerns can be overwhelming for this limited paper. In fact, topics that could be unraveled in connection to the technology is admittedly thick that Steven LaValle (2017), in a comprehensive book on virtual reality, tackled the density of the field by segmenting the nature of the technology according to various layers of issues: its science and engineering, the mathematics involved in its configuration, its dynamics and architectonics, and so forth. But it is noteworthy that he held back from fully venturing into the issue of social interaction due to the complexities of its sociocultural implications. Part of this complexity is brought by the rareness of theoretical studies pertaining to the field, although philosophical correlations have started more than two decades ago (Heim, 1994). Recognizing the overwhelming underpinnings this study of immersive technology presents, this paper limits itself within the concerns of visual language pertaining to moving images, particularly the descriptivist grammar introduced by the "frameless" $360^{\circ}$ visual capture and projection.

It is interesting to note that a sociocultural approach in the study of immersive technology, a domain falling under the study of human-computer interaction, must be given importance as the design of such technologies are being done by technological experts such as engineers and computer scientists who have little educational background that would equip them to deal with sociocultural issues (Baecker \& Buxton, 1987). 
Notwithstanding the thickness of the subject study and despite its juvenescence, this paper serves as the first small step in the commencement of my inquiry on new technologies in moving image capture and projection. I will begin my investigation by following the route taken by Shaul Hochstein (2013), a professor of neurobiology at Edmond and Lily Safra Center for Brain Sciences (ELSC) at Hebrew University who started to venture into the field with a philosophical approach. He said that

The eye looks like a camera-it got a lens and a diaphragm and an image in the back. But if the visual system were a camera taking a picture, we would need another eye to look at that picture. Just having a camera doesn't work. That's not what vision is about. (para. 7)

Hochstein (2013) continued his exposition of the filmic simulacra of reality with the ontological question, "How do we know there really is a world?" (para. 13). He traced the answer back from the time of Plato, who gave us the allegory of the cave that tells about people's perceived derivative of reality as itself the actual reality. But isn't it that we too are simply appreciating the reflection of reality on the retina of our eye? If such is the case, then it can be said that what we see is not really reality but only the reflection of it; thus, we "don't really see reality as it is" (Barry, 2013, para. 12).

My initial academic venture in the cinematic dimension of immersive technology has yielded three major insights related to the language of digitally captured moving images and its dissemination: (1) the $360^{\circ}$ camera challenges the myth ${ }^{3}$ of the filmic frame; (2) these challenges entail the reconfiguration of the idea of the kineikonic chronotope, that is, spacetime relationships in moving images of immersive visual media; (3) this reconfiguration has the potential to establish a dialogic communication during an immersive experience. For brevity's sake, only the first and the third of these insights are elaborated on in this paper.

This paper understands the place of ImT in the technological spectrum of visual cultures and uses the dialogic concept of Mikhail Bakhtin (1981) in deciphering the historiographic dialogue between imagistic mediums from the theatrical/acting performance on the dramatic/theatrical stage to its current digital immersive forms, such as virtual reality. Although the notion of dialogism was conceptualized for literature, I believe that it can be used to establish the dialogue between the past, present and future of ImT. In literature, dialogic literary works carry a continual dialogue with other works of literature and other authors. It informs and is continually informed by the previous works, not as a matter of influence but of dialogue 
that extends both ways. In other words, the previous works of literature are as altered by the dialogue as the present ones. This notion was echoed by T.S. Eliot (2015) who proposed that the present alters the past as the past directs the present.

These same principles apply to language: every word carries a history of usage unto which the word seeks to respond, while future linguistic configurations are also anticipated upon by the current usage of the word. This dialogic correlation in the literary languages is carried on in Andrew Burn's (2013) visual language dialogism between the proscenium of the theater and the frame of the cinema, the latter anticipated by the former, the former directing the latter, with both altering each other. Furthermore, the multimodal mixing desk_editing and filming soft/hardware-and its allied multimedia dissemination technologies such as cinema, television, and smartphones, are informed - and at the same time are continually informing themselves-by the past and present technologies of visual inscription, in anticipation of future trends in the field. However, the "myth of the frame" is introduced in the analysis of immersive media as the experience of $360^{\circ}$ virtual environment challenges the supremacy of the frame, a major syntactic element in the language of flat-screen cinema ${ }^{4}$ (Stewart, 2007).

The dialogic of kineikonic technology, from the trajectory of virtual reality, is best summarized by Myron Krueger (1993): "Virtual reality constitutes a new form of human experience-one that may as important to the future as film, theater, and literature have been to the past" (p. vi).

Based on these correlations between the dialogic of literature and language, in relationship with the concept of the frame, Bakhtin's dialogic also helps reveal the contrasts and progressions of the visual frame from the proscenium of the theater to the cinema, including all other iteration of the cinematic projection like projection mapping. Furthermore, current trends in ImT like camera 360 and VR gadgets tend to alter the role of the proscenium and the frame in various directions. For instance, in contrast to the present constraint set by the frame in cinematic media, the 360 technologies have given media producers and consumers the opportunity to free themselves from the tyranny of the frame, resulting in the power of the frame becoming a myth. As such, future cinematic media must anticipate the "frameless" narrative.

\section{The Proscenium: Dichotomizing Realities}

The present electronic and/or digital format of motion capture and projection has its romantic beginnings in the theatrical stage, "often seen as a naive use of the fixed camera in deference to the theatrical frame, a proxy for the eye of the audience member in the centre of the stalls" (Burn, 2013, p. 
1). Although the idea of the theatre as cinema manqué was disputed by Ben Brewster and Lea Jacobs (1998) in terms of the theater's play of the point of view, the theater's affinity with the language of the cinema is illustrated in the dialogic of the frame.

The spatial frame is primarily defined by the proscenium of the classical theater, making it akin to the frame of the television or the movie screen. More than just defining the boundaries of the mise-en-scène, the proscenium is "culturally cognate with all kinds of viewing aperture which frame the viewed object on the one hand, and the viewer's gaze on the other" (Burn, 2013, p. 1). In the temporal aspect, the shifts in time, location, scenes, and acts also serve as framing devices. But in consideration to the brevity of this paper, I will mostly concentrate on the spatial framing or proscenium.

The role of the proscenium in setting the dichotomy between the world of the spectator-the audience-and the world of fictional narrativediegesis (Danesi, 2009) - is an idea that can be readily understood from its mere presence in the theater as an architectural apparatus. It separates the physical boundary between the audience space or the real world and the set onstage or the fictional world. However, the space where the spectators can watch the actors portray their fictional lives on stage (e.g. town plaza, living room) is not limited by the physical framing of the proscenium. The dialogues between the actors, as well as offstage sound, serve as referents for an extended world that, though not visible within the viewing boundaries of the proscenium, exists in the mind of the spectator. For me, this is the appeal of classical theater: like a printed book, it leaves much to the imagination. But the human proclivity for tangible realism pushes the boundaries of the proscenium. Naturalism in 19th to early 20th-century theater and drama, for example, would require a lantern to be incorporated in the set design to serve as source of stage lighting: a lamppost or a full moon for the outdoor scene; sunlight coming in through the window for indoor scene. Then there is the forum theater of Augusto Boal and the theater in the round attempting to knock down the proscenium, in the process incorporating the audience presence into the narrative (Maeda, 2016).

Perhaps the best display of rebellion against the limitations of the proscenium is John Krizanc's play, Tamara (1981), about the Polish artist Tamara de Lempicka. The play is staged in a mansion, sometimes an armory or some edifice that can be converted into an opulent residential space where characters are portrayed to live their lives. Mel Gussow (1987) explained the concept of the play this way:

Events occur in a dozen rooms, on several staircases and in various passageways of the armory, part of which has been redesigned for the occasion. Scenes are performed 
simultaneously, which means that theatergoers choose what they think they want to see. One can follow a single character or proceed tag-style from character to character. Periodically, plots collide. Return visits to the play are encouraged, but it is possible to comprehend Tamara in one standing, especially if one communicates with other theatergoers. The show demands an openness on the part of the audience. (para. 3)

As in the theatrical innovations achieved in Tamara, various staging techniques and styles have already been invented, many of which feature theatrical staging without the proscenium. These advances in theater arts make use of the idea of physical embodiment, which benefited from the introduction of newer technologies (El Din Saad Badr, 2012). These innovations in the staging of a performance could account for the dialogic of immersive experience between cinema and dramatic theater.

\section{Cinema: Frames in Suture}

The supremacy of the frame extends from the dramatic theater to the age of the cinema, either in the temporal or the spatial aspect, as both art forms have shared principles in visual methodologies (Rose, 2002). However, the technological dexterity of the cinema has enabled it to transcend some of the limitations of the frame and even to achieve some significant degree of modifications of the frame and the framing techniques, all for the benefit of the filmic narrative. Nonetheless, the cinematic art cannot divorce itself from the preconditions of the frame, for what is cinema but a series of framed actions in a timeline, or what Tarkovsky (1984) described as mosaic made of time. Be that as it may, the quest towards a more immersive experience of the simulacrum of moving images continues to provide fulfillment.

One way of simulating the immersive experience in cinema is by "dissolving the frame" from the spectator's sight, that is, steering them toward not thinking of the existence of the camera that does the seeing or looking for them, and therefore, does the storytelling (Silverman, 1989). This system is called suture, a "filmic grammar and syntax, incorporating the spectator as signifier within a system of 'signifiers,' producing meaning while simultaneously instilling and establishing a sense of subject-hood, which is to say, the effect of suture produces the phenomenon of spectator as 'subject"' (Magrini, 2006, para. 2).

Brillante Mendoza's 2007 film Tirador [Slingshot] (Esguerra, Lapuz \& Brillante, 2007) uses suture to immerse the audience in the poverty and crime-stricken environment of Quiapo in Manila, Philippines. Although the recording equipment used in the image capture is the usual single camera 
for flat-screen projection, the camera movement is jarring, mimicking the real-world capability for panning of the spectator's eyes. Typical of this movie are shots where the camera follows a character traversing the filthy corridors of the slums and intermittently pans from side to side to get a glimpse of the environment-much like how a person would do when walking down an alley. In the process, the spectator transcends objectivizing what he watches and instead becomes part of the cinema's subject. When he becomes its subject, he settles into the cinema's experience as one of its signifiers, immersing in its diegesis as he sizes up the narrow streets; notices an open septic tank; beholds the web of power cables above him; turns away from a wall plastered with election campaign posters; notes the street children at play across him; and maneuvers a gamut of urban clichés around him. To reinforce the incorporation of offscreen scenes into a $360^{\circ}$ experience, the audio plays the role of the catalytic element in recreating the urban cacophony, which includes the bellowing sermon of the priest inside the church, the howling of the vehicles, and the murmurs in the crowd. The visual and the audio, all in splintered frames, are sutured together like a mosaic through a system of filmic techniques utilized by the filmmaker either during production or postproduction. But the final suturing agent is the spectator, who supplies whatever is missing in between the gaps of the frames, to recreate the flat-screen cinema experience and turn it into a $360^{\circ}$ appreciation of seamless sights and sounds by imagining what was not there, what was outside the frame. At this juncture, it can be said that the ultimate suturing of frames occurs in the mind of the spectator.

\section{Immersive Technology: The Frameless Experience}

Immersive technology, constituted by the operational relationships between $360^{\circ}$ image capture and virtual reality frameless projection, is still considered at its infancy that little academic study has been made in terms of its social and cultural impact (Mandal, 2013) or humanistic focus (Spicheva, 2014). The lack of such studies is brought by the fact that potential theoretical investigation of sociocultural dimension related to the technology is limited only by the actual advancement it has achieved as demonstrated by its accessibility and utility in mainstream society (LaValle, 2017). It goes without saying that the theoretical and philosophical inquiries such as the work of Heim (1994), made in the outset of the technology more than a couple of decades ago, have to be revisited in consideration of the current scenario in new literacies (Leander, 2008).

Impressive as it may seem, immersive visual technology has yet to prove, in general, its advantages over current media technologies in the arena of communication, information dissemination, and visual culture. Nonetheless, 
because of the speed of the advancements achieved by information technology, the humanistic study of $\operatorname{ImT}$ as a social phenomenon must catch up (LeMahieu, 2011). Engineers and scientists who are themselves involved in the development of the technology recognize the significance of such study. They believe that parallel undertakings between the building of a better machine and the collection of knowledge on human perception or cognition are the key to perfecting both the technology and its human utility (LaValle, 2017), over and above its current use in entertainment or leisure.

Prior to its popular use, ImT was technically unstable and expensive (Mandal, 2013). With the advent of commercially viable VR equipment and cheaper $360^{\circ}$ video camera, immersive media has found itself freely shared in social media and video websites, resulting in a revolution in the connectedness among individuals in online communities (Heim, 1994). Offline, it has been applied to various purposes, from education and information dissemination, to art appreciation and entertainment (Marrins, 2016). It has found itself in the service of curatorial historiography, creating a digital memory of the past through the archiving, curating, and archeological modeling of historical edifices and artifacts (LeMahieu, 2011). Its visual characteristic has been utilized in making big data conveniently comprehensible and interactive. Its ability to create and recreate virtual worlds made it ideal for the chaotic worlds of military simulation. With its virtual personages, that is, avatar and surrogates, it has become a useful aid for individuals with difficulty interacting with real worlds, such as those suffering from dementia due to Alzheimer's, or autism, by offering them the benefits of mediated lives (Chan, 2014).

The most obvious commonality between the $\operatorname{ImT}$ and the rest of the moving image mediums previously discussed is their kineikonic characteristics, particularly images moving in time and space. This commonality makes ImT a medium that unfolds life's drama in time and space (Alexander, 2007), a domain in visual culture dominated by the flatscreen cinema for much of the centuries since the invention of moving image capture and projection. Over and above its cinematic character, ImT is a visual medium characterized by the suppression of the frame, thus, making it the virtual simulacrum of the $360^{\circ}$ real world. Furthermore, it offers a controlled and virtually constructed environment that can be configured according to the immediate needs of individual users. Because of its inspirational effectiveness (Huntsman, 2014), it has been considered a tool in aiding individuals with impaired memory or those suffering from other disorders (Garcia, Kartolo, \& Méthot-Curtis, 2012); an instrument for early detection of the disorder (Elvey, 2016); or a venue for recreating 
the experience of dementia sufferers for non-sufferers to understand the victims of the disease (Griffiths, 2016). Research experiments as far back two years ago have been conducted in this field, but more work has yet to be done (Garcia-Betances, Waldmeyer, Fico, \& Cabrera-Umpierrez, 2015), at least one coming from a humanistic perspective.

Dialogically directed by the theatrical stage and the cinema, $\operatorname{ImT}$ goes beyond the limitations of the proscenium, the suture, and the frame of the screen towards realizing man's ageless resolve to transcend his human limitations of being confined to the laws of the natural world. ImT, acting as an extension of the mind, enhances the individual's capacity to imagine and to therefore achieve, through visual mediation, what he or she fails to accomplish due to the obstacles of human physicality.

\section{Synthesis and Conclusion}

Like any other visual medium of moving image narrative, virtual reality is a diegetic medium (Bell, 2000). Much of this is shown by its prehistory, which this paper has truncated in its discussion of the kineikonic dialogism between the theatrical stage and the cinema, including the brief archiving of the ImT's utility in the arts, entertainment, academe, and healthcare. Limited and sweeping these discussions are, what I demonstrated is a preamble to presenting the challenge of ImT-as a visual medium-towards the tyranny of the frame, with the indication that the complexity of its history is accounted by its being a radical technology (Chan, 2014). In turn, the technology's radicalism is also accounted for by the human disposition to create a simulacrum of a more immersive visual experience that is beyond his natural faculties or beyond what physical realities can afford him.

Beyond what has been demonstrated as examples in the discussion of kineikonic dialogism, the display of human undertakings in defying the supremacy of the frame can be observed in the various ways technology has enabled man to soar above flat-screen visual experience or at least attempted to: the illusion of two-dimensionality in a one-dimensional visual media such as one afforded by trompe l'oeil in painting; the panoramic apps in mobile phones; the dome of a planetarium with its semi- $360^{\circ}$ projection, a predecessor of virtual reality semi-caves; the enormous screen of the IMAX theater with its three-dimensional visual projection, and its more tactile permutation in 4Dx cinema; the computer-generated images in simulation chambers used by the military and airline training; the harmless choice to die and live again in virtual spaces provided by gaming platforms; the projection mapping of images on water fountain sprays such as those introduced in Disneyland's Phantasmic; and literally the wings afforded to man by miniature cameras mounted on drones that renders an almost 
$360^{\circ}$ capture of an expansive landscape. These applications that facilitate immersive experience have redeemed $\operatorname{ImT}$ from its pessimistic repute either as a rival to human intelligence or as a social aberration, spawned by the dystopic worldview in popular culture such as the Cyborg (1989), the Lawnmower Man (1992), The Matrix (1999), and Surrogate (2009).

From these surveys of technological materials pertinent to $\operatorname{ImT}$, the implications of a $360^{\circ}$ image capture, image projection in virtual reality, and their promise of immersive experience usher in a complex network of debates that spans an equally complex network of trajectories: political, ethical, industrial, academic, and so forth. In the midst of these complexities surrounding the subject of this paper, one realization can be deduced: ImT empowers humanity to represent abstract realities by transcending physical embodiment.

Given that ImT is part of an expanding universe of visual language, this paper started the discussion on the technology by positing that immersive technology could possibly be replaced by another much more advanced form of visual medium. However, by laying out a kineikonic dialogism involving the dramatic theater, the cinema, and virtual reality, a two-part single implication of the position has been unveiled that answers the questions formulated at the beginning of this reflection.

One part of the implication states that ImT is not just part of an expanding universe in visual culture; rather, it is the culmination of a linguistic struggle to express visual simulacra as nearest to real-world experience as possibleand expression that is immersive, embodied, $360^{\circ}$, and independent of the constraints of the frame. ImT is part of the diachronic grammar in visual language that started in the theatrical stage but was later altered in the cinematic medium due to the progress achieved in visual technologies. Although it could be the culmination, it is not the end. It can still progress further into independence-into mythic supremacy-from the restrictions of the frame, though presently, its operation is still facilitated by certain features of the frame. As part of the kineikonic dialogism, however, its further progression will be continually directed by its predecessors-a basic rule in the dialogic of language (Bakhtin, 1981).

The other part of the implication means that $\operatorname{ImT}$ is the expression of human desire to eclipse the limitation of physical embodiment. In the dialogic with its predecessors, the role of the mind in suturing framed realities implies that ImT augments the mind's capacity to immerse itself into a seamless experience of mediated reality. In other words, ImT is an extension of the mind. In the future, as the study on the sociocultural impact of immersive technology progresses, this second part of the implication shall cater to new debates about human-machine interaction that could 
influence how memory, identity, and agency can be approached using the visual dexterity of immersive technology. 


\section{References}

Alexander, L. (2007). Storytelling in time and space: Studies in the chronotope and narrative logic in screen. Journal of Narrative Theory, 37(1), 27.

Baecker, R., \& Buxton, W. (1987). Readings in human-computer technology: A multidisciplinary approach. San Mateo, CA: Morgan Kaufmann Publishers, Inc.

Bakhtin, M. (1981). The dialogic imagination: Four essays. M. Holquist (Ed.). (C. Emerson \& M. Holquist, Trans.). Austin, TX:The University of Texas Press.

Barry, D. (2013, March 1). In a cinematic frame of mind. Jerusalem Post. Retrieved from https://search. proquest.com/docview $/ 1316913043$ ?accountid $=47253$

Bell, P. (2000, March). Dialogic media productions and inter-media exchange. Journal of Dramatic Theory and Criticism, 41-55.

Burn, A. (2013). The kineikonic mode:Towards a multimodal approach to moving images. In C. Jewitt (Ed.), The Routledge handbook of multimodal analysis. London: Routledge.

Brewster, B., \& Jacobs, L. (1998). Theater to cinema: Stage pictorialism and the early feature film. Oxford: Oxford University Press.

Chan, M. (2014). Virtual reality: representations in contemporary media. New York: Bloomsbury.

Danesi, M. (2009). Dictionary of media and communications. Armonk, NY: M.E.Sharpe.

Eliot, T. (2015). The sacred wood: tradition and the individual talent. Retrieved from http://www.bartleby. com/200/sw4.html.

El Din Saad Badr, S. (2012). Virtual reality in theater spaces. Retrieved from http://www.cpas-egypt.com/ pdf/Sherouk_Shehab/Ms.c/VIRTUAL\%20REALITY\%20IN\%20THEATRE\%20SPACES.pdf

Elvey, S. (2016, October 8). Scientists using virtual reality for early diagnosis of Parkinson's, Alzheimer's and MS. Express. Retrieved from http://www.express.co.uk/news/world/719009/ Parkinson-sAlzheimer-s-MS-scientists-virtual-reality-early-diagnosis.

Garcia, L., Kartolo, A., \& Méthot-Curtis, E. (2012). A discussion of the use of virtual reality in dementia. C. Eichenberg (Ed.) Retrieved from http://www.intechopen.com/books/virtual-reality-inpsychological-medical-and-pedagogical-applications/a-discussion-of-the-use-of-virtual-realityin-dementia.

Garcia-Betances, R., Waldmeyer, M. A., Fico, G., \& Cabrera-Umpierrez, M. (2015, May 12). A succinct overview of virtual reality technology use in alzheimer's disease. National Center for Biotechnology Information. Retrieved from https://www.ncbi.nlm.nih.gov/pmc/articles/ PMC4428215/.

Griffiths, S. (2016, January 13). Terrying 'dementia tour' reveals what it's like to suffer from the condition: Experience uses gloves and glasses to rob people of their senses and recreate fear patients feel. MailOnLine. Retrieved from http://www.dailymail.co.uk/sciencetech/article-3397302/Terrifyingdementia-tour-reveals-like-suffer-condition-Experience-uses-gloves-glasses-rob-people-sensesrecreate-fear-patients-feel.html.

Gussow, M. (1987, December 3). The stage: 'Tamara'. The New York Times. Retrieved from http://www. nytimes.com/1987/12/03/theater/the-stage-tamara.html.

Heim, M. (1994). The metaphysics of virtual reality. Oxford: Oxford University Press.

Hochstein, S. (2013, March). In B.Davis, In a cinematic frame of mind. elsc.huji.ac.il. Retrieved from https:// elsc.huji.ac.il/sites/default/files/baw2013jpost.pdf 
Huntsman, M. (2014, April 3). How a virtual reality forest helps Alzheimer's patients. Alzheimers.net. Retrieved from http://www.alzheimers.net/2014-04-03/how-a-virtual-reality-forest-helpsalzheimers-patients/.

Maeda, H. (2016). Forum theatre in a community theatre environment. Retrieved from https://oda.hioa. no/en/item/asset/dspace:10493/Maeda.pdf.

Krueger, M. (1993). Foreword. In M. Heim, The metaphysics of virtual reality. Oxford: Oxford University Press.

LaValle, S. (2017). Virtual reality. Cambridge: Cambridge University Press.

LeMahieu, D. L. (2011). Digital memory, moving images, and the absorption of historical experience. Film \& History: An Interdisciplinary Journal, 41(1), 82-106.

Lapuz, F. (Producer), Jover, R. (Writer), \& Mendoza, B. (Director). (2007). Tirador [Motion picture]. Philippines: Peccadillo Pictures (UK).

Leander, K. (2008). Toward a connective ethnography of online/offline literacy network. In J. Coiro, M. Knobel, C. Lankshear, \& D. Leu (Eds.), Handbook of research on new literacies (pp. 33-65). New York: Routledge.

Magrini, J. (2006, February 11). On the system of the "suture" in cinema. Otherzine. Retrieved from http:// othercinema.com/otherzine/archives/index.php?issueid=15\&article_id=33.

Mandal, S. (2013, April). Brief introduction of virtual reality \& its challenges. International Journal of Scientific \& Engineering Research, 4(4), 304-309.

Marrins, K. (2016, June 24). Engage your givers with virtual reality - a starter guide. The Guardian. Retrieved from https://www.theguardian.com/voluntary-sector-network/2016/jun/24/givers-virtual-realityguide-charities-raise-funds

Rose, G. (2002). Visual methodologies: An introduction to the interpretation of visual materials. London: SAGE Publications.

Silverman, K. (1989). The subject of semiotics. New York: Oxford University Press.

Spicheva, D. (2014). Image communication in virtual reality culture. International Journal of Architectural Theory (32), 81-90.

Stewart, G. (2007). Framed time: Towards a postfilmic cinema. Chicago: University of Chicago Press.

Tarkovsky, A. (1982). Masterclass with Andrei Tarkovsky: Cinema is mosaic made of time. Cinephelia and Beyond. Retrieved from https://cinephiliabeyond.org/filmmakers-masterclass-with-andreitarkovsky-cinema-is-a-mosaic-made-of-time/.

\section{Endnotes}

1 The term immersive technology or ImT, as used in this paper, encompasses both the capture (e.g., $360^{\circ}$ camera) and dissemination (e.g., virtual reality) of moving images. In some parts of the paper, specific mention of $360^{\circ}$ camera apart from virtual reality and vice versa will be articulated in order to specify references to each but still within the overarching concept pertinent to this study.

${ }^{2}$ This study differentiates between two modes of image capture and image projection. The current image projection prevalent in television and commercial movie cinema is the flat screen, which benefits from the image capture provided by the single camera. Single camera generally refers to various types of cameras regardless of aspect ratio and sensor specification. The $360^{\circ}$ camera differs from the single cam as it uses multiple cams in one single apparatus in order to capture a $360^{\circ}$ coverage of the moving 
environment. The projection, though, sometimes afforded by a flat screen, is fully experienced through "surround viewing" provided by a Head Mounted Display (HMD) or VR semi-cave (e.g., simulators).

${ }^{3}$ The filmic frame has been an essential protocol in the major constructions of film theory and study. In fact, a filmmaker cannot practically produce a film without being constrained by the frame; ontologically speaking, filmmaking (and viewing) is framing. With the introduction of the $360^{\circ}$ image capture and projection, the dominance of the frame in filmmaking and all other cinematic genres is challenged and put into question.

${ }^{4}$ For consistency, single cam image capture and/or flat screen projection will be generally referred to as flat-screen cinema in this paper, to differentiate them from the technology of $360^{\circ}$ image capture and projection, which is sometimes simply referred to as 360 .

GREGG S. LLOREN is an assistant professor at the University of the Philippines Cebu handling courses in digital scholarship, visual culture, and communications. He earned his MSc by Research (Merit) in Interdisciplinary Creative Practices at the University of Edinburgh, UK, working on visual language and social semiotics. He is currently pursuing his doctoral studies in the same university working on immersive technology, specifically virtual reality for the reconstruction of impaired memory, such as dementia of the Alzheimer's type. (Corresponding author: gslloren@up.edu.ph) 